\title{
Prognostic value of hepatitis B viral infection in patients with nasopharyngeal carcinoma in the intensity-modulated radiotherapy era
}

\author{
Shuo-Han Zheng ${ }^{1,2 \#}$, Shu Zhou ${ }^{3 \#}$, Guan-Nan Wang ${ }^{1,4}$, Zi-Lu Huang ${ }^{1,2}$, Song-Ran Liu ${ }^{1,5}$, Chen Chen ${ }^{1,2}$, \\ Ya-Lan Tao ${ }^{1,2}$, Hui Chang ${ }^{1,2}$, Shi-Rong Ding ${ }^{1,2}$, Ruo-Nan Liao ${ }^{6}$, Chang Chen ${ }^{6}$, Yun-Fei Xia ${ }^{1,2}$ \\ ${ }^{1}$ State Key Laboratory of Oncology in South China, Collaborative Innovation Center for Cancer Medicine, Guangdong Key Laboratory of \\ Nasopharyngeal Carcinoma Diagnosis and Therapy, Sun Yat-sen University Cancer Center, Guangzhou, China; ${ }^{2}$ Department of Radiation \\ Oncology, Sun Yat-sen University Cancer Center, Guangzhou, China; ${ }^{3}$ Department of Radiotherapy, The first affiliated hospital of Nanjing Medical \\ University, Nanjing, China; ${ }^{4}$ Department of Head \& Neck Surgery, Sun Yat-sen University Cancer Center, Guangzhou, China; ${ }^{5}$ Department of \\ Pathology, Sun Yat-sen University Cancer Center, Guangzhou, China; ${ }^{6}$ Zhongshan Medical College, Sun Yat-sen University, Guangzhou, China \\ Contributions: (I) Conception and design: SH Zheng, S Zhou, YF Xia; (II) Administrative support: C Chen, YL Tao, H Chang, YF Xia; (III) Provision \\ of study materials or patients: S Zhou, YF Xia; (IV) Collection and assembly of data: SH Zheng, S Zhou, GN Wang, ZL Huang, RN Liao, C Chen; (V) \\ Data analysis and interpretation: SH Zheng, SR Liu, C Chen, YL Tao, H Chang, SR Ding, YF Xia; (VI) Manuscript writing: All authors; (VII) Final \\ approval of manuscript: All authors. \\ \#These authors contributed equally to this work. \\ Correspondence to: Yun-Fei Xia. Department of Radiation Oncology, Sun Yat-sen University Cancer Center, State Key Laboratory of Oncology in South \\ China, Collaborative Innovation Center for Cancer Medicine, 651 Dongfeng Road East, Guangzhou 510060, China. Email: xiayf@sysucc.org.cn.
}

Background: Whether hepatitis B virus (HBV) infection poses risk to patients with nasopharyngeal carcinoma (NPC) in the intensity-modulated radiotherapy (IMRT) era remains unclear.

Methods: 953 patients with non-metastatic, newly diagnosed NPC who received detection of serologic hepatitis B surface antigen (HBsAg) and treated with IMRT were retrospectively reviewed. 171 patients had HBV infection (HBsAg seropositive). Propensity score matching method (PSM) and stabilized inverse probability of treatment weighting (IPTW) were used to address confounding. The survival rates were evaluated by Kaplan-Meier analysis and the survival curves were compared by Log-rank test. Prognostic factors were explored by multivariate analysis.

Results: No significant survival differences were observed between HBsAg-negative group and HBsAgpositive group [5-year overall survival (OS), $87.7 \%$ vs. $83.9 \%, \mathrm{P}=0.181$; locoregional recurrence-free survival (LRFS), $83.5 \%$ vs. 78.3\%, $\mathrm{P}=0.109$; distant metastasis-free survival (DMFS), 80.2\% vs. 77.9\%, $\mathrm{P}=0.446$; progression-free survival (PFS), $77.4 \%$ vs. $71.4 \%, \mathrm{P}=0.153$ ], consistent with the results of PSM and IPTW analysis. Further analyses revealed that $\mathrm{HBV}$ infection was an independent prognostic factor for poor OS [multivariate analysis; hazard ratio (HR), 3.74; 95\% confidence interval (CI), 1.45-9.68; $\mathrm{P}=0.006$ ], LRFS (HR, 2.86; 95\% CI, 1.37-5.95); $\mathrm{P}=0.005$ ] in patients with stage $\mathrm{N} 1$, DMFS (HR, 2.65; 95\% CI, 1.15-6.09; $\mathrm{P}=0.022$ ) and $\mathrm{PFS}(\mathrm{HR}, 2.63 ; 95 \% \mathrm{CI}, 1.34-5.14 ; \mathrm{P}=0.005)$. Among HBsAg-positive patients, liver protection improved OS (90.3\% vs. $77.2 \%$; $\mathrm{P}=0.022)$.

Conclusions: HBV infection is an independent risk factor for patients with stage N1 NPC in the IMRT era. Hepatic protection may benefit the survival of $\mathrm{HBsAg}$-positive patients.

Keywords: Nasopharyngeal carcinoma (NPC); intensity-modulated radiotherapy (IMRT); hepatitis B virus (HBV); prognosis

Submitted Jul 19, 2021. Accepted for publication Oct 07, 2021.

doi: $10.21037 /$ tcr-21-1340

View this article at: https://dx.doi.org/10.21037/tcr-21-1340 


\section{Introduction}

Nasopharyngeal carcinoma (NPC) is a malignant tumor of the nasopharyngeal epithelium, whose aetiological factor is properly the infection of Epstein-Barr virus (EBV) (1). In endemic regions, especially in South China, the world age-standardized incidence rate of NPC can be up to 25.39/100,000 person-years (2). Another endemic virus in South China is the hepatitis B virus (HBV), where 8\% to $15 \%$ of people were found to have positive hepatitis $\mathrm{B}$ virus surface antigen (HBsAg) (3). More than one study suggested that HBsAg-positive patients with NPC had a poorer prognosis than $\mathrm{HBsAg}$-negative in the endemic area. Liu et al. demonstrated hepatitis B viral (HBV) infection was an independent prognostic factor for worse overall survival (OS), locoregional recurrence-free survival (LRFS), progression-free survival (PFS) significantly and distant metastasis-free survival (DMFS) marginally (4). Xu et al. found that worse outcomes and distant-failure especially liver metastasis were more common in HBsAg-positive patients (5). However, Weng et al. found that no statistically significant difference in OS, disease-free survival (DFS), LRFS or DMFS in patients with different HBsAg status (6). Whether the $\mathrm{HBV}$ infection is an independent risk factor or not is controversial.

Radiotherapy (RT) is the primary and only curative treatment for NPC due to its special location and high radiosensitivity. Intensity-modulated radiotherapy (IMRT) is preferred if modern radiation technology is available (1). At present, IMRT is applied to all the NPC patients in our hospital. The 5-year LRFS, PFS and OS of IMRT were about $90 \%, 80 \%$ and $85 \%$ respectively, significantly higher than that of the 2-dimensional (2-D) technique (7). However, the most common RT technique mentioned in previous papers was 2 -D RT, nearly $40 \%$ of patients were treated with that (4-6). And RT techniques were confirmed to be an independent prognostic factor in OS, PFS, LRFS (4). Thus, whether the survival of HBsAg-positive patients with NPC is still poorer than those with HBsAg-negative in the IMRT era remains unknown. We conducted a retrospective study to explore the prognostic effect of $\mathrm{HBV}$ infection with the propensity score-based analyses to reduce possible biases to a minimum. We present the following article in accordance with the STROBE reporting checklist (available at https://dx.doi.org/10.21037/tcr-21-1340).

\section{Methods}

\section{Study population}

We continuously assessed the medical records of HBsAgpositive patients with NPC treated at the Sun Yat-sen University Cancer Center (Guangzhou, China). The eligibility criteria for this study were: (I) biopsy-proven NPC; (II) newly diagnosed M0 stage patients; (III) treated with IMRT; (IV) plasma EBV DNA load before treatment (EBV-DNA) was measured; (V) serologic markers HBsAg was tested; (VI) without other malignant diseases. According to the above criteria, $782 \mathrm{HBsAg-negative} \mathrm{patients} \mathrm{from}$ January 1, 2008 to December 31, 2010, and 171 HBsAgpositive patients from January 1, 2008 to December 31, 2011 were consecutively included. The study was conducted in accordance with the Declaration of Helsinki (as revised in 2013). The study was approved by ethics board of Sun Yat-sen University Cancer Center (No. B2021-206-01) and individual consent for this retrospective analysis was waived.

\section{Treatment}

All patients received IMRT. Prescribed radiation doses were $\geq 66$ Gy to the primary tumor and $\geq 50$ Gy to the bilateral cervical lymph nodes and potential sites of local infiltration. Patients were treated with $30-35$ fractions with 5 daily fractions per week for 6 to 7 weeks. Induction, concomitant or adjuvant chemotherapy was applied to patients. The selection of chemotherapy regimen and the number of corresponding cycles were determined by clinicians. The platinum-based chemotherapy regimen was used extensively. Prophylactic antiviral therapy would be administered if the patients had HBV replication (a serum HBV DNA $>0$ IU/mL) and/or liver injury before or during treatment. Hepatic protection was mainly used in patients with hepatic dysfunction, which was dependent on hepatic function tested every 7 or 14 days and estimated according to the National Cancer Institute - Common Terminology Criteria for Adverse Events (NCI-CTCAE) version 4.0 score system.

\section{Data collection and follow-up}

Demographic, clinical and follow-up data were collected with uniform database templates from our hospital information system by two physicians specializing in 
NPC. Any disagreements were resolved by consensus. Sex, age, diagnostic time, treatment data, restaged tumor, node, metastasis (TNM) classification based on the 8th edition of the Union for International Cancer Control/ American Joint Committee on Cancer staging system were obtained. Blood tests for $\mathrm{HBV}$ and $\mathrm{EBV}$ infection performed before the treatment for NPC. Briefly, HBsAg was detected quantitatively or qualitatively by collecting blood samples, separating serum, and then measuring serum samples by chemiluminescence immunoassay, electrochemiluminescence immunoassay or enzyme-linked immunosorbent assay. EBV-DNA was measured using real-time quantitative polymerase chain reaction assay targeting the BamH I-W region of the EBV genome (details were described in the Appendix file). The liver function, including alanine aminotransferase (ALT), aspartate aminotransferase (AST) and total bilirubin (TBIL), was measured from biochemical studies. Treatment outcomes were ascertained from follow-up medical clinic records of the hospital information system, or contacting the patients or their families by telephone, and/or in the outpatient clinic every 3 to 6 months during the first 3 years and every 6 to 12 months thereafter (or until death). Survival status, local failure and distant metastasis were recorded according to clinical symptoms, physical examination or photographic results at every follow-up visit. The last follow-up date was May 31, 2017.

\section{Statistical analysis}

Statistical analyses were carried out with Statistical Product and Service Solutions software version 24.0 (SPSS Inc., Chicago, IL, USA) and R Statistical Software version 3.2.0 (Foundation for Statistical Computing, Vienna, Austria). All analyses were two-tailed and the significance level was specified as $\mathrm{P}<0.05$. The follow-up duration was calculated from the date of diagnosis to the date of death from any cause or censored at the last visit or last follow-up date of May 31, 2017. Four commonly used survival endpoints, including OS, LRFS, DMFS and PFS, were assessed. OS was defined as the time from diagnosis to death from any cause. LRFS was defined as the time from diagnosis to the date of the first locoregional failure or death from any cause, whichever came first. DMFS was defined as the time from diagnosis to the date of the first distant failure or death from any cause, whichever came first. PFS was defined as the time from diagnosis to the date of disease progression or death from any cause, whichever came first. Variables including age (divided into two groups by median age 44 years old; range, 11 to 78 years old), gender, histological type, $\mathrm{T}$ classification, $\mathrm{N}$ classification and clinical stage were introduced in a logistic regression to compute a propensity score for every patient. A propensity score matching method (PSM) was employed to match the patients from the HBsAg-positive group to the HBsAg-negative group, using the $1: 2$ nearest neighbor technique with a small caliper of 0.05 to ensure better balance. And a stabilized inverse probability of treatment weighting (IPTW) was calculated with the estimated propensity scores (8). The chi-square test or Fisher's exact test was used to compare categorical variables between two groups. Survival curves were plotted using the Kaplan-Meier method with the log-rank test. Subgroup analyses were conducted in the matched cohort and HBsAg-positive patients. Multivariate analysis was performed using the Cox proportional hazards model.

\section{Results}

\section{Baseline characteristics of the patients}

Among 953 patients included in this study, 171 patients were seropositive for HBsAg. With the use of PSM, $162 \mathrm{HBsAg-}$ positive patients and $324 \mathrm{HBsAg-negative} \mathrm{patients} \mathrm{were}$ selected. EBV-DNA was cut off by 1,500 copies/mL $(5,9)$. The baseline characteristics of patients were summarized in Table 1. HBV infection was more common in older or male patients, as well as in keratinizing histology. After matching and weighting with the propensity score, host, tumor and treatment-related factors were balanced in two groups (all $\mathrm{P}>0.05$ ).

\section{Effect of HBV infection in patients with NPC}

Patterns of treatment failure are summarized in Table 2. The median follow-up time for the entire cohort was 81 months (range, 1-112 months). Up to the last day of follow-up, the overall survival rate was more than $80 \%$. Patients with locoregional failure were more frequent in $\mathrm{HBsAg}$-positive group than HBsAg-negative group, 25.6\% compared to $18.3 \%$ weighted by IPTW $(\mathrm{P}=0.028)$. The occurrence of distant metastasis with different sites was similar in these two groups. And the difference of treatment failure was not significant.

Survival analyses were shown in Figures 1-3. The 5-year OS, LRFS, DMFS and PFS of HBsAg-negative patients 
Table 1 Baseline characteristics of the patients with nasopharyngeal carcinoma

\begin{tabular}{|c|c|c|c|c|c|c|c|c|c|}
\hline Characteristics & \multicolumn{3}{|c|}{ Unmatched, n (\%) } & \multicolumn{3}{|c|}{ PSM, n (\%) } & \multicolumn{3}{|c|}{ IPTW, \% } \\
\hline Age (year) & & & $<0.001$ & & & 0.947 & & & 0.777 \\
\hline$\leq 44$ & $375(48.0)$ & $107(62.6)$ & & $199(61.4)$ & 99 (61.1) & & 50.5 & 49.3 & \\
\hline Male & $554(70.8)$ & $137(80.1)$ & & $258(79.6)$ & $129(79.6)$ & & 72.5 & 71.7 & \\
\hline Female & $228(29.2)$ & $34(19.9)$ & & $66(20.4)$ & $33(20.4)$ & & 27.5 & 28.3 & \\
\hline WHO pathology & & & 0.009 & & & 0.727 & & & 0.074 \\
\hline T classification & & & 0.476 & & & 0.915 & & & 0.465 \\
\hline $\mathrm{T} 1$ & $55(7.0)$ & $10(5.8)$ & & $23(7.1)$ & $9(5.6)$ & & 6.9 & 6.8 & \\
\hline $\mathrm{T} 2$ & $169(21.6)$ & $29(17.0)$ & & $60(18.5)$ & $29(17.9)$ & & 21.2 & 17.3 & \\
\hline T3 & $335(42.8)$ & $81(47.4)$ & & $151(46.6)$ & $79(48.8)$ & & 42.7 & 49 & \\
\hline $\mathrm{T} 4$ & $223(28.5)$ & $51(29.8)$ & & $90(27.8)$ & $45(27.8)$ & & 29.2 & 26.9 & \\
\hline $\mathrm{N}$ classification & & & 0.978 & & & 0.784 & & & 0.795 \\
\hline No & $89(11.4)$ & $18(10.5)$ & & $33(10.2)$ & $13(8.0)$ & & 11.3 & 9.3 & \\
\hline ॥ & $115(14.7)$ & $20(11.7)$ & & $40(12.3)$ & $19(11.7)$ & & 14.4 & 11.8 & \\
\hline III & $374(47.8)$ & $86(50.3)$ & & $155(47.8)$ & $84(51.9)$ & & 47.6 & 53.6 & \\
\hline IV & $279(35.7)$ & $63(36.8)$ & & $120(37.0)$ & 57 (35.2) & & 36.3 & 33.3 & \\
\hline EBV-DNA & & & 0.677 & & & 0.335 & & & 0.460 \\
\hline$<1,500$ copies $/ \mathrm{mL}$ & $375(48.0)$ & 79 (46.2) & & $157(48.5)$ & $71(43.8)$ & & 48.1 & 45.0 & \\
\hline$\geq 1,500$ copies $/ \mathrm{mL}$ & $407(52.0)$ & $92(53.8)$ & & $167(51.5)$ & $91(56.2)$ & & 51.9 & 55.0 & \\
\hline Chemotherapy & & & 0.744 & & & 1.000 & & & \\
\hline No & $108(13.8)$ & $22(12.9)$ & & $42(13.0)$ & $21(13.0)$ & & 13.6 & 13.7 & \\
\hline Yes & $674(86.2)$ & $149(87.1)$ & & $282(87.0)$ & $141(87.0)$ & & 86.4 & 86.3 & \\
\hline
\end{tabular}

Table 1 (continued) 
Table 1 (continued)

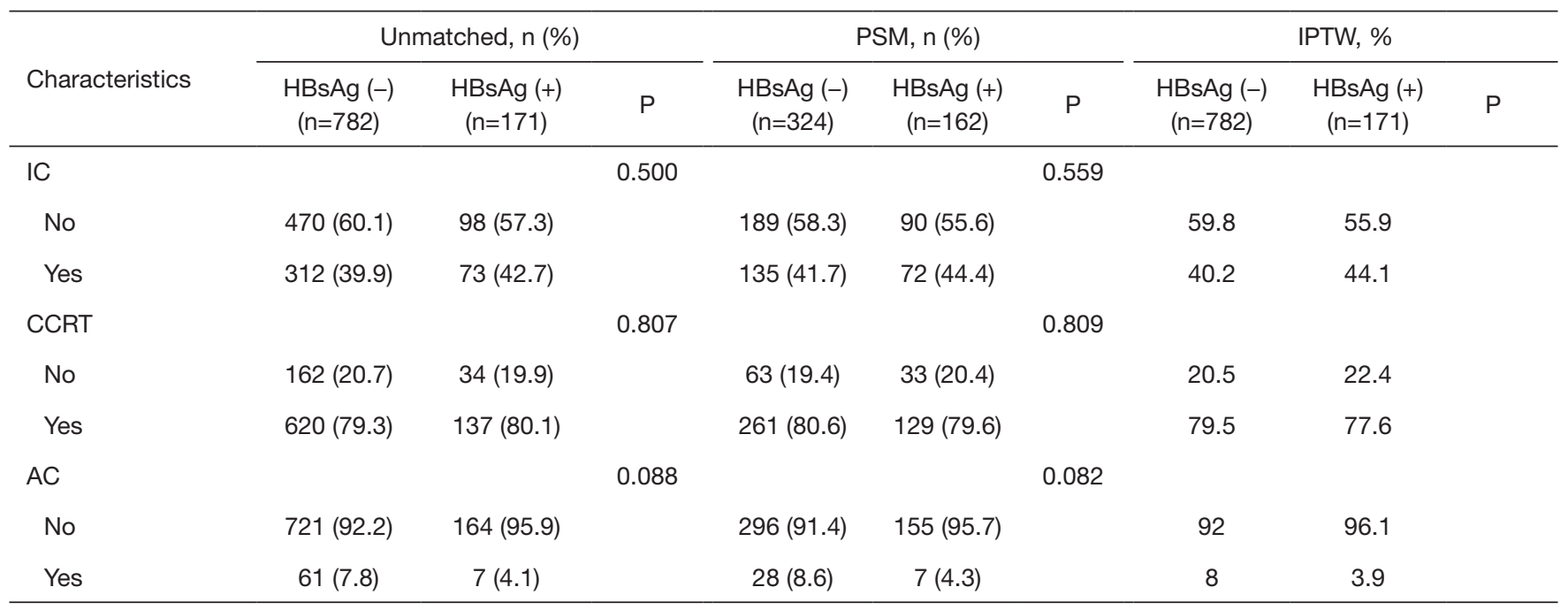

PSM, propensity score matching; IPTW, inverse probability of treatment weighting; HBsAg, hepatitis B surface antigen; HBsAg (-), HBsAgnegative; HBsAg (+), HBsAg-positive; WHO, World Health Organization; TNM, tumor, node, and metastasis; EBV-DNA, pre-treatment plasm Epstein-Barr virus DNA load; IC, induction chemotherapy; CCRT, concurrent chemoradiotherapy; AC, adjuvant chemotherapy.

Table 2 Patterns of treatment failure

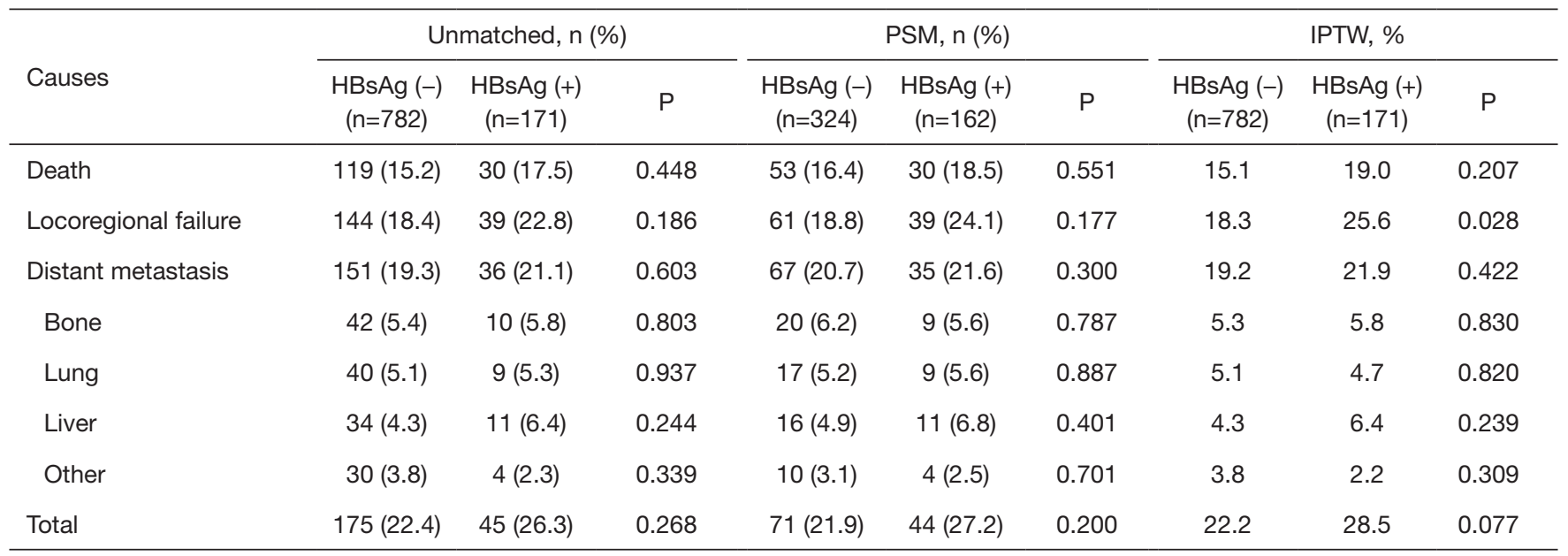

PSM, propensity score matching; IPTW, inverse probability of treatment weighting; HBsAg, hepatitis B surface antigen; HBsAg (-), HBsAg-negative; HBsAg (+), HBsAg-positive.

were similar with HBsAg-positive patients (OS, $87.7 \%$ vs. 83.9\%; LRFS, $83.5 \%$ vs. $78.3 \%$; DMFS, $80.2 \%$ vs. $77.9 \%$; PFS, $77.4 \%$ vs. $71.4 \%$; all $\mathrm{P}>0.05$ ), consistent with the results of PSM and IPTW analysis. Then the multivariate analysis was performed to adjust for various prognostic factors of all the patients in the propensity score matched cohort in Table 3. Parameters including age, sex, pathology;
T classification, $\mathrm{N}$ classification, TNM staging, EBV-DNA, HBsAg status, chemotherapy, induction chemotherapy (IC), concurrent chemoradiotherapy (CCRT) and adjuvant chemotherapy (AC) were introduced to the model. And the results demonstrated that HBsAg was not an independent factor for any outcome, while $\mathrm{N}$ classification was an independent adverse prognostic factor for all the outcomes. 
A

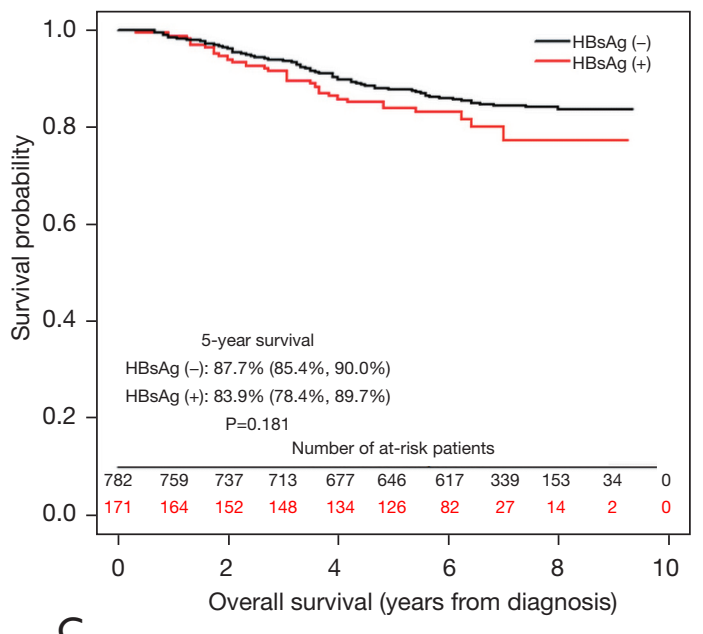

C

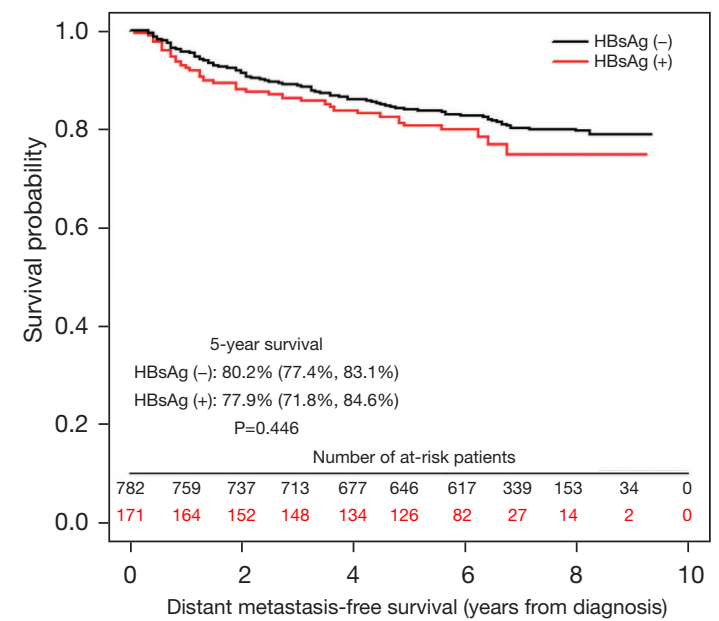

\section{B}
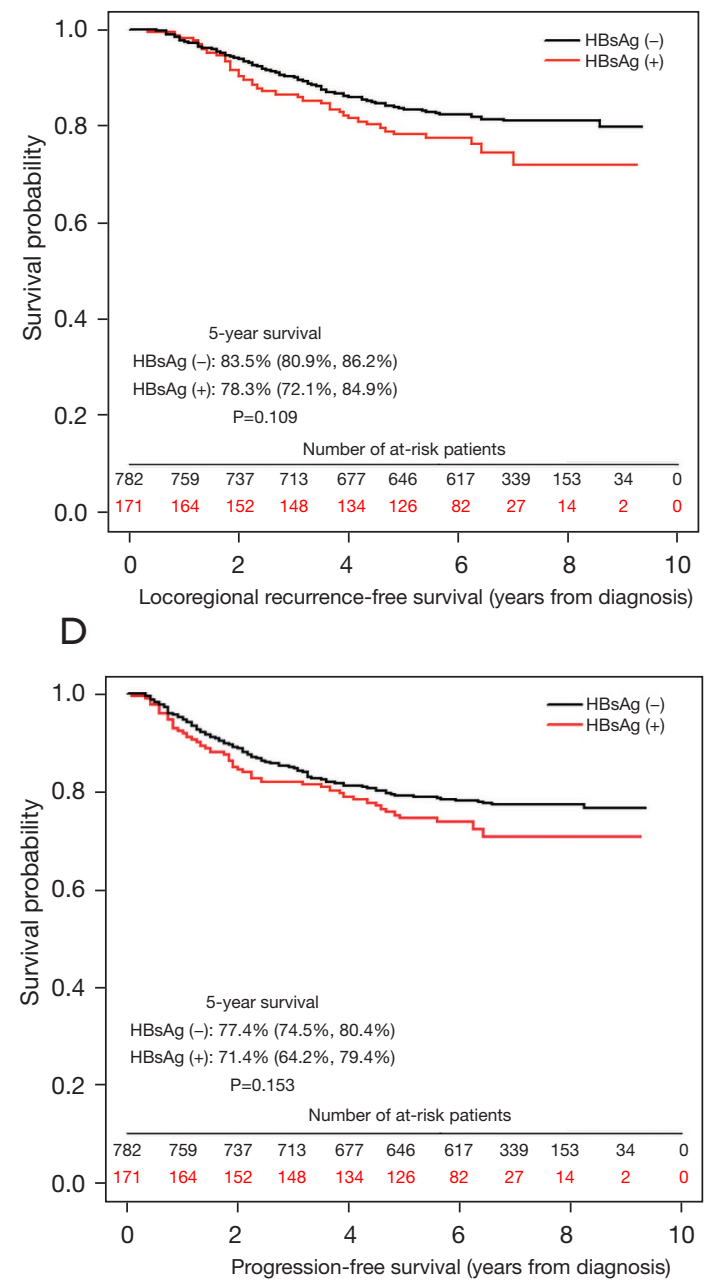

Figure 1 Kaplan-Meier estimates of overall survival (A), locoregional recurrence-free survival (B), distant metastasis-free survival (C), and progression-free survival (D) in the unmatched cohort. The 5-year survival rates and $95 \%$ CI of two groups were shown.

\section{Effect of HBV infection in patients with stage N1 NPC}

Survival analyses of patients with N1 in the propensity score matched cohort were shown in Figure 4. The 5-year OS, LRFS and PFS were poorer in HBsAg-positive patients except DMFS (OS, 82.6\% vs. 94.3\%, $\mathrm{P}=0.007$; LRFS, $75.3 \%$ vs. 89.7, $\mathrm{P}=0.004$; DMFS, $81.5 \%$ vs. $90.7 \%, \mathrm{P}=0.090$; PFS, $72.9 \%$ vs. $87.1 \%, \mathrm{P}=0.009)$. Then the multivariate analysis was performed to confirm the prognostic value of HBV infection in patients with N1. Age, sex, pathology; T classification, TNM staging, EBV-DNA, HBsAg status, chemotherapy, IC, CCRT and AC were included in the model and the results indicated that HBsAg seropositivity was an independent risk factor for OS, LRFS, DMFS and
PFS, respectively (Table 4).

\section{Effect of antiviral therapy or hepatic protection in patients with NPC and HBV infection}

The liver condition of HBV-infected patients was evaluated by ultrasound, computed tomography (CT) or positron emission tomography-computed tomography (PET-CT). No liver cirrhosis was found according to the result of the examinations. There were 62 patients treated with antiviral agents like entecavir, adefovir dipivoxil or lamivudine and 89 with hepatic protection using glutathione or diammonium glycyrrhizinate and so on. Among the patients with antiviral 
A

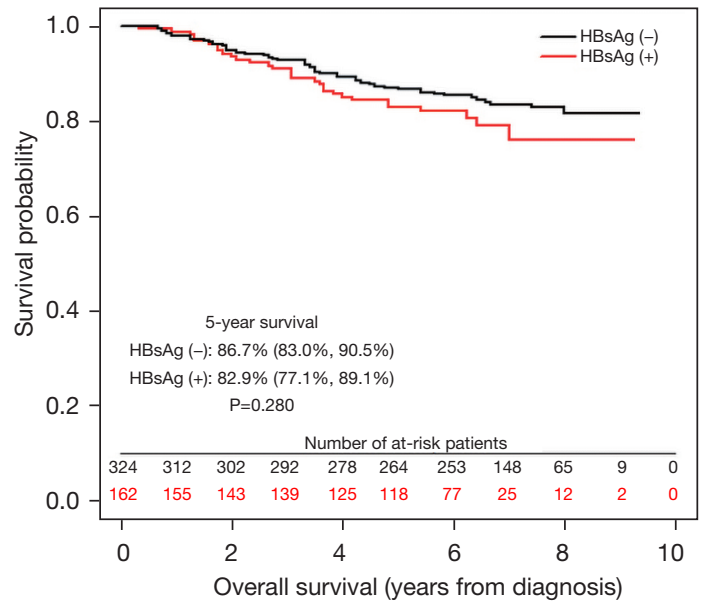

C

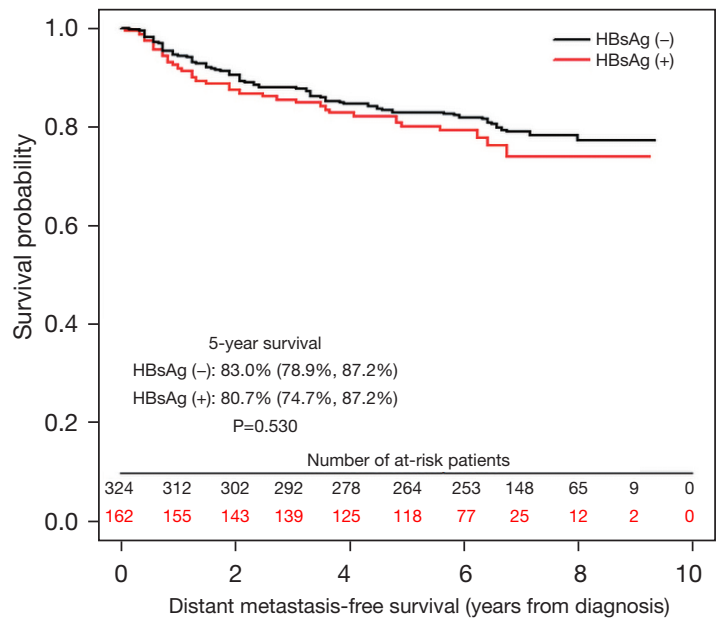

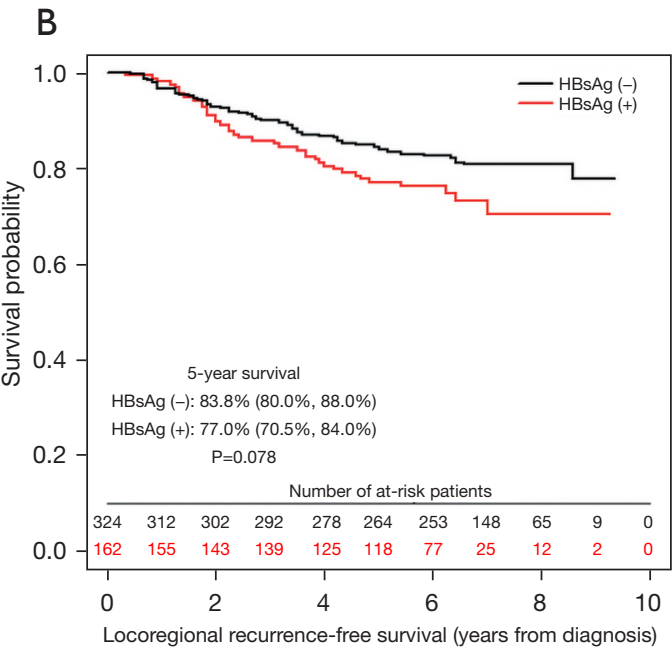

D

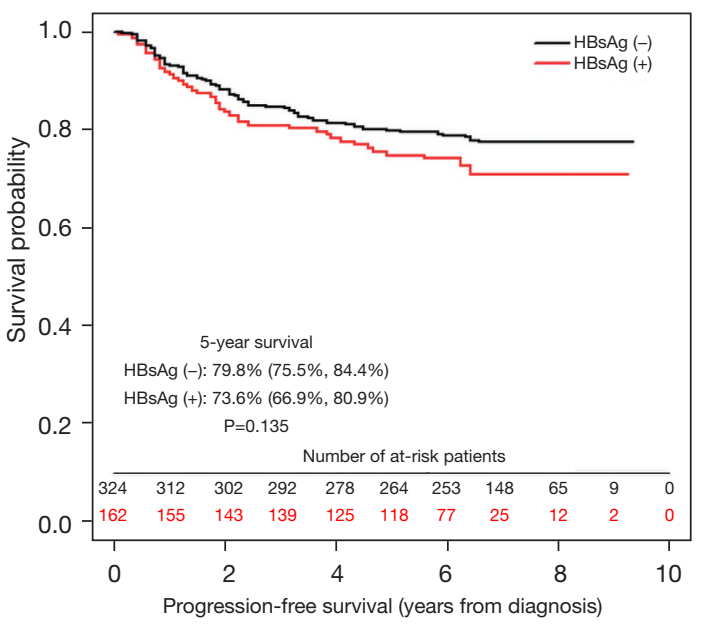

Figure 2 Kaplan-Meier estimates of overall survival (A), locoregional recurrence-free survival (B), distant metastasis-free survival (C), and progression-free survival (D) with the propensity score matched analysis. The 5-year survival rates and $95 \%$ CI of two groups were shown.

therapy, 43 patients $(69.4 \%)$ had liver dysfunction and $41(66.1 \%)$ received hepatoprotective agents. In the liver protective group, 76 patients $(85.4 \%)$ had the liver injury (53 patients with grade $1 ; 20$ with grade $2 ; 3$ with grade 3 ) and $41(46.1 \%)$ received antiviral agents. The 5 -year survival outcomes of patients with NPC and HBV infection classified by having antiviral therapy or hepatic protection during treatment were shown in Table 5. Patients with antiviral therapy had poor DMFS (85.7\% vs. $71.4 \%$; $\mathrm{P}=0.021$ ). And patients with liver protection were prone to attain higher OS (90.3 vs. $77.2 \% ; \mathrm{P}=0.022)$.

\section{Discussion}

HBsAg positivity rates were demonstrated to be higher significantly in patients with head and neck cancer (10). Several previously published studies had investigated the impact of HBV infection on the prognosis of NPC patients from an endemic area, where the majority of them were received 2-D RT $(4,5)$. IMRT has become the first choice of patients diagnosed with NPC. Therefore, the role of HBV infection in NPC that poses risk to patients treated with IMRT warrants more research. To our knowledge, this is 

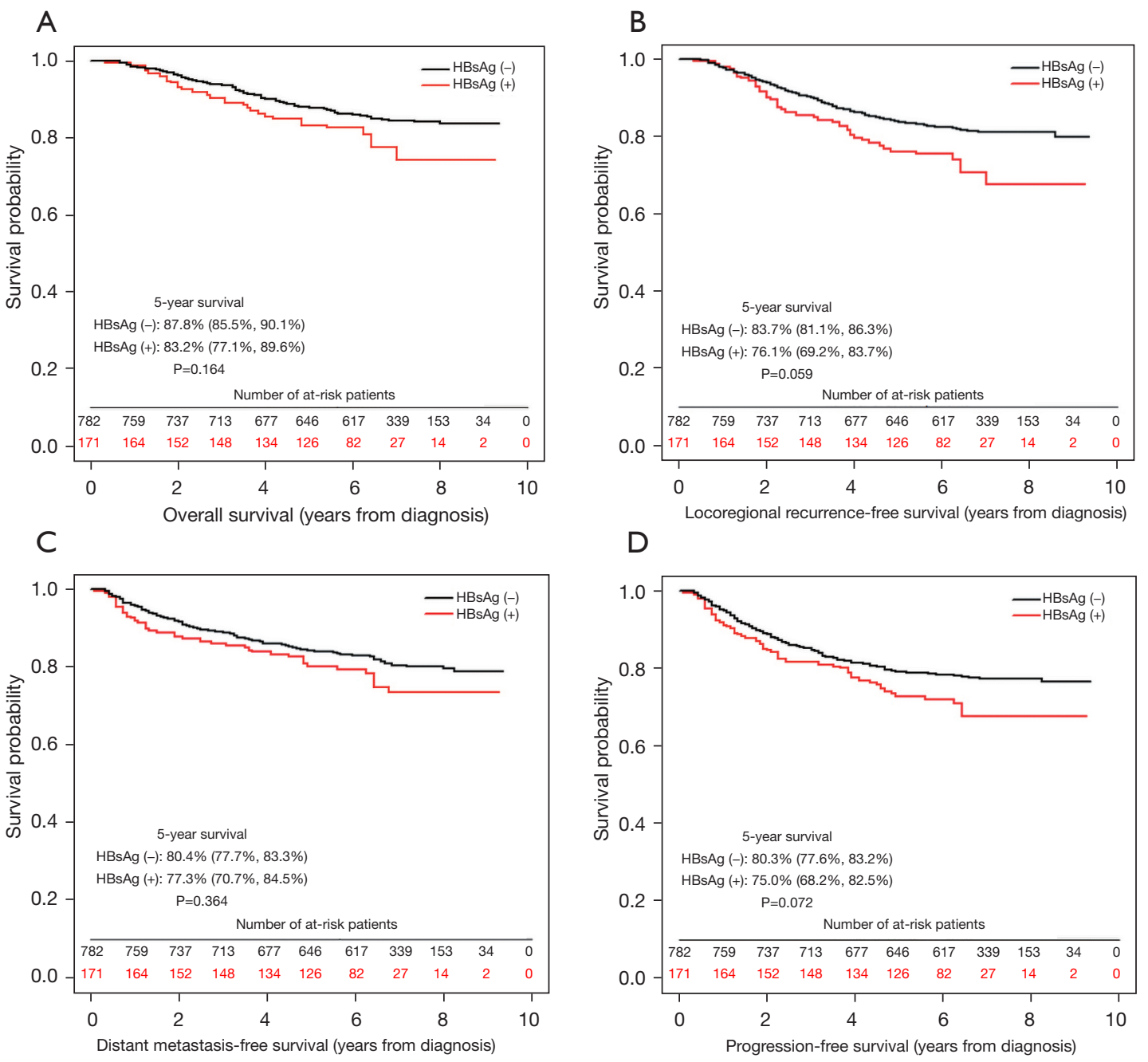

Figure 3 Kaplan-Meier estimates of overall survival (A), locoregional recurrence-free survival (B), distant metastasis-free survival (C), and progression-free survival (D) with the inverse probability of treatment weight-adjusted analysis. The 5-year survival rates and $95 \%$ CI of two groups were shown.

the first attempt to compare the survival outcomes between NPC patients with or without HBV infection treated with IMRT. PSM and IPTW were adapted based on the factors that could affect the prognosis like age, gender, histological type, TNM category and clinical stage, attenuating the bias as much as possible. Moreover, the influence of antiviral therapy or hepatic protection among HBV-infected patients with NPC was explored in the present study.

The data indicated HBsAg-positive patients with NPC had similar outcomes as those with HBsAg-negative status in the IMRT era, inconsistent with the previous studies. Several reasons might account for them. In our study, the only used RT technique was IMRT, which could improve survival rates significantly like local failure-free rate, distant failure-free rate, disease-specific survival and OS especially in stage III-IV (11). 87.1\% of HBsAg-positive patients in our study were staging III-IV, which indicated that the prognosis of NPC patients might be improved by the evolution of the RT technique even with $\mathrm{HBV}$ infection. Chemotherapy was also prevalent in our study. The baseline characteristics showed there was no significant difference in systemic therapy between these two groups and $86.2 \%$ of HBsAg-negative patients received cytotoxic drugs and $79.3 \%$ underwent CCRT. In Xu's study, only $73.4 \%$ of patients with $\mathrm{HBV}$ infection were treated with CCRT, significantly lower than $98.8 \%$ of patients in the 
Table 3 Multivariate analyses of 5-year survival outcomes in the propensity score matched cohort

\begin{tabular}{|c|c|c|c|c|c|c|c|c|}
\hline Subgroup & \multicolumn{2}{|l|}{ OS } & \multicolumn{2}{|l|}{ LRFS } & \multicolumn{2}{|l|}{ DMFS } & \multicolumn{2}{|l|}{ PFS } \\
\hline Age & $1.70(1.04-2.80)$ & 0.035 & $1.66(1.07-2.57)$ & 0.023 & - & - & - & - \\
\hline $\mathrm{N}$ classification & $1.68(1.14-2.49)$ & 0.009 & 1.52 (1.06-2.19) & 0.022 & $1.88(1.30-2.74)$ & 0.001 & $1.61(1.16-2.25)$ & 0.005 \\
\hline TNM staging & $2.16(1.07-4.40)$ & 0.033 & - & - & - & - & - & - \\
\hline IC & $0.50(0.28-0.86)$ & 0.013 & - & - & $0.55(0.33-0.91)$ & 0.020 & - & - \\
\hline
\end{tabular}

OS, overall survival; LRFS, locoregional relapse-free survival; DMFS, distant metastasis-free survival; PFS, progression-free survival; HR, hazard ratio; Cl, confidence interval; TNM, tumor, node, and metastasis; EBV-DNA, pre-treatment plasm Epstein-Barr virus DNA load; IC, induction chemotherapy.
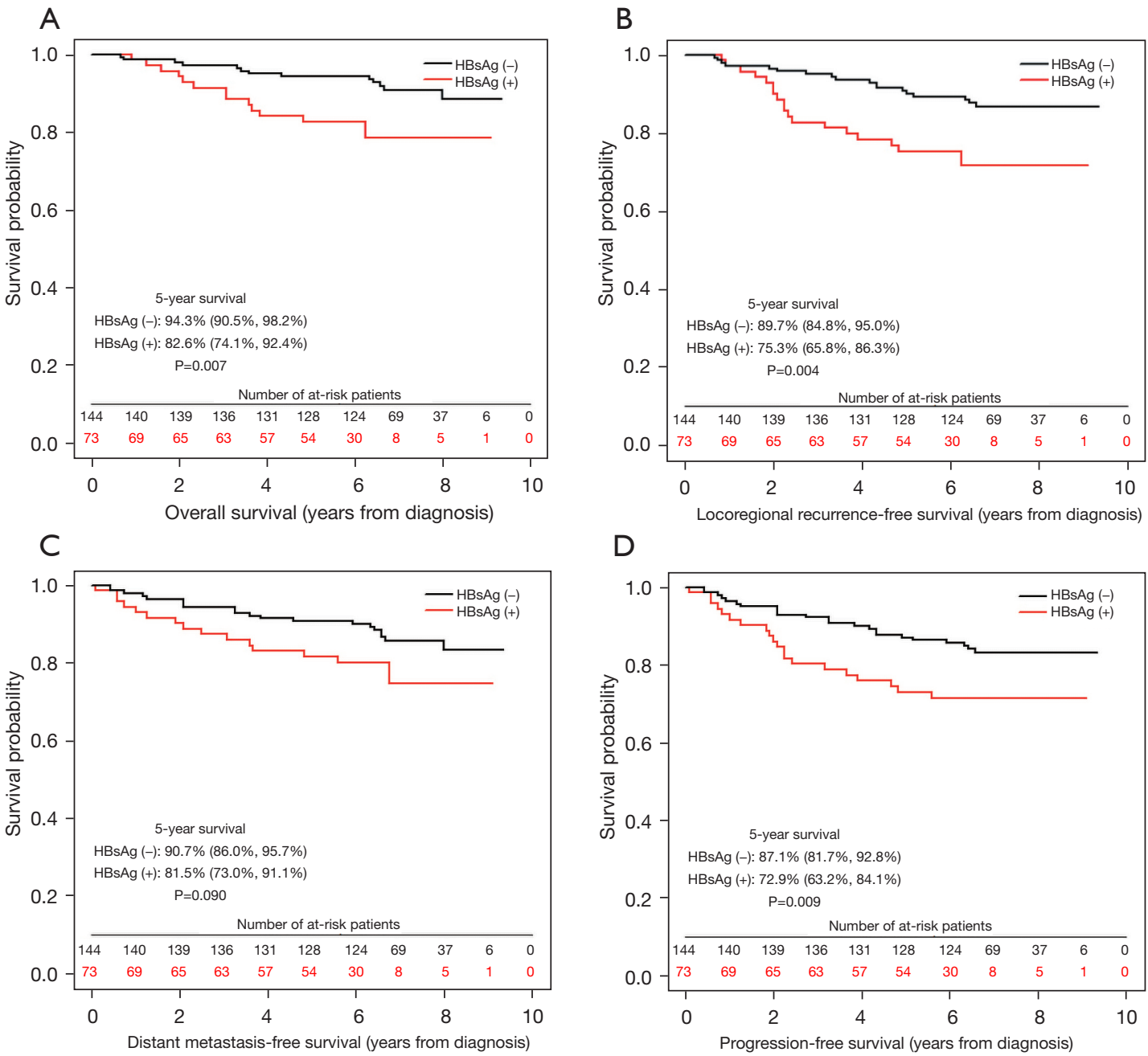

Figure 4 Kaplan-Meier estimates of overall survival (A), locoregional recurrence-free survival (B), distant metastasis-free survival (C), and progression-free survival (D) of patients with N1 in the propensity score matched cohort. The 5 -year survival rates and $95 \%$ CI of two groups were shown. 
Table 4 Multivariate analyses of 5-year survival outcomes of patients with N1 in the propensity score matched cohort

\begin{tabular}{|c|c|c|c|c|c|c|c|c|}
\hline Subgroup & \multicolumn{2}{|l|}{ OS } & \multicolumn{2}{|l|}{ LRFS } & \multicolumn{2}{|l|}{ DMFS } & \multicolumn{2}{|l|}{ PFS } \\
\hline Age & $2.85(1.02-7.99)$ & 0.046 & & & & & & \\
\hline EBV-DNA & $3.20(1.03-9.92)$ & 0.044 & & & $3.02(1.22-7.46)$ & 0.017 & $2.03(1.00-4.13)$ & 0.050 \\
\hline HBsAg status & $3.74(1.45-9.68)$ & 0.006 & $2.86(1.37-5.95)$ & 0.005 & $2.65(1.15-6.09)$ & 0.022 & $2.63(1.34-5.14)$ & 0.005 \\
\hline
\end{tabular}

OS, overall survival; LRFS, locoregional relapse-free survival; DMFS, distant metastasis-free survival; PFS, progression-free survival; HR, hazard ratio; $\mathrm{Cl}$, confidence interval; TNM, tumor, node, and metastasis; EBV-DNA, pre-treatment plasm Epstein-Barr virus DNA load; HBsAg, hepatitis B surface antigen.

Table 5 Survival analyses of patients with nasopharyngeal carcinoma and hepatitis B infection

\begin{tabular}{|c|c|c|c|c|c|c|c|c|}
\hline Outcomes & \multicolumn{4}{|c|}{ Antiviral therapy } & \multicolumn{4}{|c|}{ Hepatic protection } \\
\hline OS & $86.4 \%$ & $79.5 \%$ & $1.63(0.75-3.51)$ & 0.212 & $77.2 \%$ & $90.3 \%$ & $0.39(0.17-0.90)$ & 0.022 \\
\hline LRFS & $80.6 \%$ & $74.2 \%$ & $1.40(0.72-2.74)$ & 0.320 & $76.1 \%$ & $80.5 \%$ & $0.75(0.38-1.45)$ & 0.385 \\
\hline DMFS & $85.7 \%$ & $71.4 \%$ & $2.22(1.11-4.45)$ & 0.021 & $74.9 \%$ & $85.9 \%$ & $0.52(0.25-1.06)$ & 0.068 \\
\hline
\end{tabular}

*, P values were calculated by the log-rank test. OS, overall survival; LRFS, locoregional relapse-free survival; DMFS, distant metastasisfree survival; PFS, progression-free survival; HR, hazard ratio; $\mathrm{Cl}$, confidence interval.

HBsAg-negative group (5). Many studies confirmed that the addition of concomitant chemotherapy to radiotherapy significantly improved survival outcomes of locoregionally advanced NPC $(12,13)$. Maybe we should take this factor into account when analyzing the survival difference between HBsAg-positive and HBsAg-negative groups.

$\mathrm{HBV}$ infection was proved to be an independent adverse prognostic factor in patients with early-stage (stage I/ II) NPC or locoregionally advanced (stage III/IV) NPC $(4,6)$. However, differences of survival outcomes between HBsAg-positive group and HBsAg-negative group were not significant in patients with early-stage or locoregionally advanced NPC in the current study (Table S1). Instead, we found that HBV infection was an independent risk factor in patients with N1 NPC, but the underlying reason needed further research. $\mathrm{Xu}$ et al. reported that no correlation between pretreatment plasma EBV DNA load and HBsAg status, but the survival rates were significantly lower for patients with both HBV and EBV infection, compared with negative patients (5). In the study, we found that patients with EBV-DNA $\geq 1,500$ copies/mL in the HBsAgpositive group had worse LRFS and PFS (Table S2), but the multivariate analyses failed to confirm the independent adverse impact of HBV and revealed that EBV-DNA was an independent prognosticator of OS and LRFS (Table 3). The EBV-DNA was considered to be correlated with the tumor load and was suggested as a screening, prognostic, and surveillance factor or therapeutics reference in NPC (14-19). Whether HBV existed or not, EBV DNA was still a powerful biomarker as long as the tumor cell was not eliminated.

Another intriguing finding in our study was that the application of liver protective drugs improved the OS of HBV-infected patients. Chemotherapy contributed to $\mathrm{HBV}$ reactivation and liver injury in $\mathrm{HBsAg}$-positive patients with cancer, and antiviral drug like lamivudine was demonstrated to decrease HBV reactivation and its related negative outcomes (such as hepatitis and chemotherapy disruption and mortality) in chemotherapeutic patients with $\mathrm{HBV}$ infection $(20,21)$. In our study, nearly half of the liver protective patients had antivirus drugs and most of them have minor liver dysfunction. Patients were more likely to finish the course of chemotherapy if treated with liver protection. The liver was important in metabolism and immunity. Qiu et al. found that activation of liver-associated immunity due to $\mathrm{HBV}$ infection reduced the incidence 
of liver metastasis in patients with colorectal cancer and elevated the surgical resection rate of liver metastatic lesions (22).

Some limitations couldn't be ignored in our study. The sample size of patients with HBV infection was small and we wasted much information on HBsAg-negative patients even we use the 1:2 nearest neighbor technique. Secondly, it was a retrospective study, so the occurrence of antiviral therapy, hepatic dysfunction and hepatic protection was only collected from medical records. Besides, HBV DNA was not monitored in every HBsAg-positive patient and we hardly distinguished whether the abnormal liver function was caused by HBV reaction or hepatotoxicity of chemotherapy drugs. And the prophylactic antiviral therapy was not administrated routinely in HBsAg-positive patients in the current study. Thus, the findings of this study warranted further research with a larger population.

\section{Conclusions}

$\mathrm{HBV}$ infection is an independent unfavorable factor for patients with stage N1 NPC in the IMRT era. Antivirus therapy and hepatic protection may benefit the survival of HBsAg-positive patients.

\section{Acknowledgments}

Funding: This work was supported by the National Key R \& D Program of Precise Medical Research of China (No. 2016YFC0904600), the National Natural Science Foundation of China (No. 81872464), and the Natural Science Foundation of Guangdong Province (No. 2018A030310236).

\section{Footnote}

Reporting Checklist: The authors have completed the STROBE reporting checklist. Available at https://dx.doi. org/10.21037/tcr-21-1340

Data Sharing Statement: available at https://dx.doi. org/10.21037/tcr-21-1340

Conflicts of Interest: All authors have completed the ICMJE uniform disclosure form (available at https://dx.doi. org/10.21037/tcr-21-1340). The authors have no conflicts of interest to declare.
Ethical Statement: The authors are accountable for all aspects of the work in ensuring that questions related to the accuracy or integrity of any part of the work are appropriately investigated and resolved. The study was conducted in accordance with the Declaration of Helsinki (as revised in 2013). The study was approved by ethics board of Sun Yat-sen University Cancer Center (No. B2021-20601 ) and individual consent for this retrospective analysis was waived.

Open Access Statement: This is an Open Access article distributed in accordance with the Creative Commons Attribution-NonCommercial-NoDerivs 4.0 International License (CC BY-NC-ND 4.0), which permits the noncommercial replication and distribution of the article with the strict proviso that no changes or edits are made and the original work is properly cited (including links to both the formal publication through the relevant DOI and the license). See: https://creativecommons.org/licenses/by-nc-nd/4.0/.

\section{References}

1. Chua MLK, Wee JTS, Hui EP, et al. Nasopharyngeal carcinoma. Lancet 2016;387:1012-24.

2. Wei KR, Zheng RS, Zhang SW, et al. Nasopharyngeal carcinoma incidence and mortality in China, 2013. Chin J Cancer 2017;36:90.

3. Lv JW, Chen YP, Huang XD, et al. Hepatitis B virus screening and reactivation and management of patients with nasopharyngeal carcinoma: A large-scale, big-data intelligence platform-based analysis from an endemic area. Cancer 2017;123:3540-9.

4. Liu X, Li X, Jiang N, et al. Prognostic value of chronic hepatitis $\mathrm{B}$ virus infection in patients with nasopharyngeal carcinoma: analysis of 1301 patients from an endemic area in China. Cancer 2014;120:68-76.

5. Xu T, Huang $Z$, Deng $Y$, et al. Clinical implications of hepatitis B viral infection in Epstein-Barr virus-associated nasopharyngeal carcinoma. J Clin Virol 2015;64:64-71.

6. Weng JJ, Wei JZ, Li M, et al. Effects of hepatitis B virus infection and antiviral therapy on the clinical prognosis of nasopharyngeal carcinoma. Cancer Med 2020;9:541-51.

7. Sun X, Su S, Chen C, et al. Long-term outcomes of intensity-modulated radiotherapy for 868 patients with nasopharyngeal carcinoma: an analysis of survival and treatment toxicities. Radiother Oncol 2014;110:398-403.

8. Austin PC, Stuart EA. Moving towards best practice when 
using inverse probability of treatment weighting (IPTW) using the propensity score to estimate causal treatment effects in observational studies. Stat Med 2015;34:3661-79.

9. Lin JC, Wang WY, Chen KY, et al. Quantification of plasma Epstein-Barr virus DNA in patients with advanced nasopharyngeal carcinoma. N Engl J Med 2004;350:2461-70.

10. Kocoglu H, Karaca M, Tural D, et al. Hepatitis B and $\mathrm{C}$ rates are significantly increased in certain solid tumors: A large retrospective study. J Cancer Res Ther 2018;14:S774-8.

11. Lee AW, $\mathrm{Ng}$ WT, Chan LL, et al. Evolution of treatment for nasopharyngeal cancer--success and setback in the intensity-modulated radiotherapy era. Radiother Oncol 2014;110:377-84.

12. Blanchard P, Lee A, Marguet S, et al. Chemotherapy and radiotherapy in nasopharyngeal carcinoma: an update of the MAC-NPC meta-analysis. Lancet Oncol 2015;16:645-55.

13. Ribassin-Majed L, Marguet S, Lee AWM, et al. What Is the Best Treatment of Locally Advanced Nasopharyngeal Carcinoma? An Individual Patient Data Network MetaAnalysis. J Clin Oncol 2017;35:498-505.

14. Yip TT, Ngan RK, Fong AH, et al. Application of circulating plasma/serum EBV DNA in the clinical management of nasopharyngeal carcinoma. Oral Oncol 2014;50:527-38.

15. Zhang J, Peng H, Li WF, et al. Individualized induction chemotherapy by pre-treatment plasma Epstein-Barr viral DNA in advanced nasopharyngeal carcinoma. BMC Cancer 2018;18:1276.

16. Chan KCA, Woo JKS, King A, et al. Analysis of Plasma

Cite this article as: Zheng SH, Zhou S, Wang GN, Huang ZL, Liu SR, Chen C, Tao YL, Chang H, Ding SR, Liao RN, Chen C, Xia YF. Prognostic value of hepatitis B viral infection in patients with nasopharyngeal carcinoma in the intensitymodulated radiotherapy era. Transl Cancer Res 2021;10(11):46244635. doi: $10.21037 /$ tcr-21-1340
Epstein-Barr Virus DNA to Screen for Nasopharyngeal Cancer. N Engl J Med 2017;377:513-22.

17. Lin JC, Wang WY, Liang WM, et al. Long-term prognostic effects of plasma epstein-barr virus DNA by minor groove binder-probe real-time quantitative PCR on nasopharyngeal carcinoma patients receiving concurrent chemoradiotherapy. Int J Radiat Oncol Biol Phys 2007;68:1342-8.

18. Tang LQ, Chen QY, Fan W, et al. Prospective study of tailoring whole-body dual-modality 18Ffluorodeoxyglucose positron emission tomography/ computed tomography with plasma Epstein-Barr virus DNA for detecting distant metastasis in endemic nasopharyngeal carcinoma at initial staging. J Clin Oncol 2013;31:2861-9.

19. Chan KC, Chan AT, Leung SF, et al. Investigation into the origin and tumoral mass correlation of plasma EpsteinBarr virus DNA in nasopharyngeal carcinoma. Clin Chem 2005;51:2192-5.

20. Xu Z, Dai W, Wu YT, et al. Prophylactic effect of lamivudine on chemotherapy-induced hepatitis B virus reactivation in patients with solid tumour: A meta-analysis. Eur J Cancer Care (Engl) 2018;27:e12799.

21. Zhang LL, Zhou GQ, Li YC, et al. Induction Chemotherapy Has No Prognostic Value in Patients with Locoregionally Advanced Nasopharyngeal Carcinoma and Chronic Hepatitis B Infection in the IMRT Era. Transl Oncol 2017;10:800-5.

22. Qiu HB, Zhang LY, Zeng ZL, et al. HBV infection decreases risk of liver metastasis in patients with colorectal cancer: A cohort study. World J Gastroenterol 2011;17:804-8. 